\title{
Opiniones de los profesionales sanitarios acerca de la definición de trastorno mental grave
}

\author{
Opinions of healthcare professionals on the definition \\ of severe mental illness
}

\section{J.A. Martínez Larrea}

\section{Sr. Director:}

He leído con mucho interés el estudio cualitativo sobre las opiniones de los profesionales del ámbito sanitario acerca de la definición del trastorno mental grave publicado en su revista ${ }^{1}$. Como bien señalan los autores el concepto de trastorno mental grave se desarrolla como una herramienta para la mejor gestión y organización de los recursos de salud mental, facilitando un uso más racional de los mismos en función de las necesidades de los pacientes. Si bien es cierto, como también señalan los autores, que hasta el momento no se han establecido criterios sólidos y uniformes, ni existe un acuerdo internacional que defina un trastorno mental grave, no es menos ciertos que dicho concepto ha estado presente en la planificación estratégica, en la organización y en el funcionamiento de muchos recursos y organizaciones de Salud Mental desde hace casi 40 años.

En Navarra el concepto se incluye por primera vez en las memorias de salud mental de 1998 y se mantiene hasta la actuali$\mathrm{dad}^{2}$. Es un momento en el que se intenta reorganizar la asistencia en los centros de salud mental, trascendiendo el mero diagnóstico clínico utilizado hasta ese momento. Los motivos eran evidentes. La necesidad de priorizar la atención de determinados perfiles de pacientes, facilitando su acceso y mejorando su frecuentación de los centros de salud mental ante una tendencia cada vez mayor a incrementar la atención de personas con trastornos mentales menos graves o incluso sin diagnóstico de trastorno mental que accedían de forma indiscriminada al sistema especializado de atención en salud mental. Esto se unía a un desarrollo de la red de salud mental extrahospitalaria ya entonces notable y unas prácticas de asistencia que primaban la retención de los usuarios, con escasez de altas en todo tipo de pacientes sin discriminar importancia o gravedad de los mismos. Ya entonces, muy lejos aún de los tiempos de la actual crisis, no se contemplaba la posibilidad de un crecimiento de los recursos de salud mental paralelo al incremento de la demanda por lo que se exigía una estrategia de cambio ante el riesgo evidente de perder calidad en la atención, sobre todo de las personas con afectaciones más graves.

\author{
Correspondencia: \\ J.A. Martínez Larrea \\ Director de Salud Mental. \\ Servicio Navarro de Salud-Osasunbidea \\ Plaza de la Paz, s/n. 2 $2^{\text {a }}$ Planta \\ 31002 Pamplona (Navarra) \\ Email: amartinl@cfnavarra.es
}


En esta primera fase se optó por una agrupación diagnóstica amplia de lo que en Navarra se consideró Trastorno Mental Grave (Tabla 1). Comparando a simple vista los datos de actividad de la Red de Salud Mental de Navarra en 1998 y 2013 podemos concluir que, al menos parcialmente, los objetivos se están cumpliendo. Los pacientes con trastorno mental grave atendidos en la red de salud mental superan a los pacientes con trastorno mental menos grave pese a que la demanda de primeras consultas a la red de salud mental sigue siendo mayor para trastornos mentales menos graves (Fig. 1).

Tabla 1. Diagnósticos incluidos en el programa de trastornos mentales graves de Navarra, 1998.

\begin{tabular}{l}
\hline T. mentales orgánicos \\
\hline Esquizofrenia \\
\hline T. paranoide \\
\hline T. afectivos \\
\hline Otros trastornos psicóticos \\
\hline Tentativa de suicidio \\
\hline Agorafobia con angustia \\
\hline T. Obsesivo-compulsivo \\
\hline T. Personalidad \\
\hline T. conducta alimentaria \\
\hline T. generalizados del desarrollo \\
\hline Retraso mental \\
\hline
\end{tabular}

El concepto de trastorno mental grave ha sido clave también en la Comunidad Foral de Navarra para la planificación y desarrollo del Programa de Atención al Trastorno Mental Grave ${ }^{3}$ que ha desarrollado desde el año 2005 una amplia oferta de recursos residenciales y rehabilitadores, laborales y de apoyo comunitario en el entorno social y sociosanitario específicos para este perfil de pacientes. Este programa fue objeto de valoración por parte de un grupo de expertos utilizando metodología Delphi que fue publicado en esta revista $^{4}$. En 2010 el programa fue revisado y se precisó el concepto de trastorno mental grave redefiniendo los grupos diagnósticos priorizados, el niveles de discapacidad o dependencia y una duración de al menos 2 años $^{5}$.

Para seguir progresando me parecen de extraordinaria importancia las conclusiones del trabajo publicado. Coincido plenamente en la limitada utilidad del criterio diagnóstico clínico y en especial del tiempo de evolución como determinantes de la definición de un trastorno mental grave. Del mismo modo resulta cada vez más evidente la centralidad que el criterio disfunción o discapacidad está tomando en la planificación de los recursos y la asistencia a personas con trastornos mentales. El diagnóstico y el tiempo son factores indicativos de riesgo de padecer una mayor afectación funcional y de que dicha afectación funcional resulte en mayor o menor medida irreversible. Algo tenemos que aprender en el entorno sanitario de los desarrollos realizados en el área de los servicios sociales. Para los servicios sociales es el grado de discapacidad o dependencia el que se asocia al derecho garantizado de una prestación asistencial en función de las necesidades e independientemente del diagnóstico o tiempo de evolución.

También es cierto que ha sido en el ámbito de los servicios sociales donde el criterio de ausencia o existencia de una red de apoyo familiar y/o social se ha introducido no como mecanismo específico de definición de un trastorno mental grave, sino de priorización de la atención por parte de los recursos públicos ante la existencia de recursos limitados.

Muchas son las variables que se pueden relacionar con la gravedad de un trastorno mental grave y teniendo en cuenta nuestro conocimiento de las mismas es tiempo ya de avanzar en la aplicación de tecnologías que nos permitan una más adecuada clasificación de los pacientes en función de sus necesidades contemplando toda esta diversidad de variables. Los modelos de estratificación o segmentación de la población ${ }^{6}$, ampliamente desarrollados en el campo de la salud como pilar fundamental de las estrategias de atención a pacientes crónicos, pueden ser perfectamente apli- 


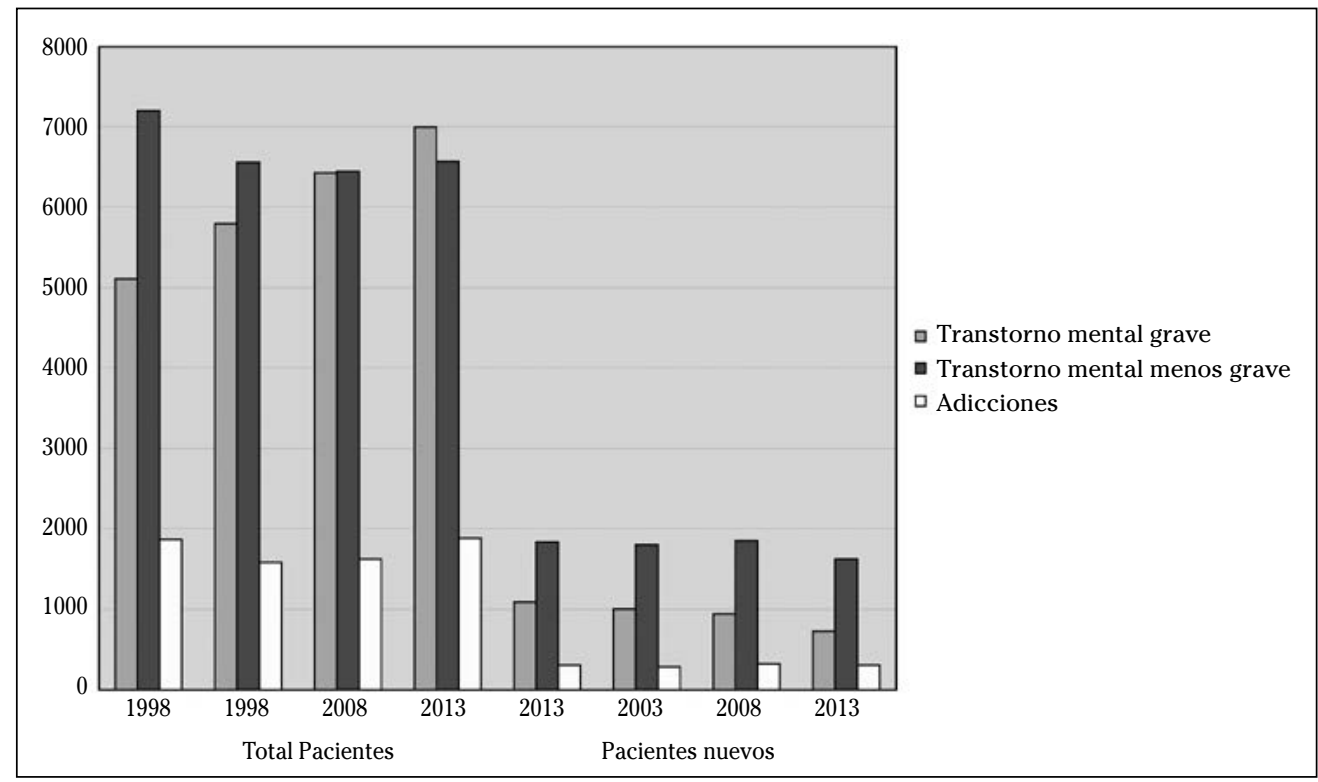

Figura 1. Pacientes menores de 16 años atendidos en los centros de Salud Mental de Navarra. 1998 y 2013 (Datos de las memorias)).

cables en nuestro campo. Utilizando herramientas de información basadas en minería de datos podremos identificar colectivos homogéneos de pacientes con patrones de necesidades y comportamiento similares. Esto nos permitirá la priorización clínica de las intervenciones sanitarias para lograr un mayor impacto en términos de resultados en salud.

Nuestra necesidad principal a corto plazo es que cada profesional asistencial pueda identificar fácilmente a los pacientes con mayor carga de enfermedad y por consiguiente con mayores necesidades asistenciales. Una vez identificados podremos diseñar nuevas modalidades de atención más eficaces y eficientes y medir sus resultados para la salud de los pacientes. A medio plazo, estos sistemas de información pueden facilitarnos la predicción de eventos (ingresos, reingresos, institucionalización, etc.) para poder intervenir precozmente en su prevención. Y a largo plazo pueden ser una herramienta muy útil para la asignación más adecuada de los recursos sanitarios.
Son muchos los aspectos en los que la salud mental ha sido pionera en la organización de la asistencia a los pacientes más graves y crónicos: hospitales de día como alternativa a la hospitalización parcial, desarrollo del espacio sociosanitario, definición de perfiles de pacientes, etc. Ahora tenemos la obligación de seguir siendo pioneros compartiendo nuestro conocimiento y aprendiendo de las aportaciones que nos llegan desde otros ámbitos.

\section{BIBLIOGRAFÍA}

1. Conejo Cero S, Moreno Peral P, Morales Asencio Jy, Alot Montes A, Garcia-Herrera JM, Gonzalez Lopez Ms et al. Opiniones de los profesionales del ámbito sanitario acerca de la definición de trastorno mental grave. Un estudio cualitativo. An Sist Sanit Navar 2014; 37: 223-233.

2. Servicio Navarro de Salud-Osasunbidea. Gobierno de Navarra. Memorias de Salud Mental de Navarra. http://www.navarra.es/home_es/Gobierno+de+Navarra/ Organigrama/Los + departamentos/Salud/Organigrama/Estructura+Organica/ Servicio+Navarro+de+Salud/Publicaciones/ Memorias/ 
3. Departamento de Bienestar Social, Deporte y Juventud. Gobierno de Navarra. Programa de Atención a Personas con Trastorno Mental Grave, 2005. http://www.navarra. es/NR/rdonlyres/1A2478B3-2651-418B-89FAB24BBA5383C0/152344/PROGRAMATMG.pdf

4. Brugos A, Arbeloa B, Astrain MV, López S, Otero M, Monreal C et al. Valoración del programa social de atención a personas con trastorno mental grave: un estudio Delphi. An Sist Sanit Navar 2007; 30: 101-112.

5. Departamento de Bienestar Social, Deporte y Juventud. Gobierno de Navarra. Reorde- nación de Programa Atención a Persona con Trastorno Mental Grave. 2010. https://www. navarra.es/NR/rdonlyres/0B760BA1-ED114F9D-B142-7B3683B6FDC0/156092/InformeReordenaciondefinitivo1.pdf

6. Departamento de Salud. Gobierno de Navarra. Modelo de Segmentación. En Estrategia Navarra de atención integrada a pacientes crónicos y pluripatológicos 2013, 67-89. http://www.navarra.es/home_es/Temas/Portal+de+la+Salud/ Ciudadania/Nuevo+modelo+asistencial/Estrat egia+navarra+de+atencion+integrada+a+pacie ntes+cronicos $+y+$ pluripatologicos/ 